\title{
Psychology and the Use of Intuitions in Philosophy
}

\author{
Brian Talbot \\ Philosophy Department, University of Colorado at Boulder
}

\begin{abstract}
There is widespread controversy about the use of intuitions in philosophy. In this paper I will argue that there are legitimate concerns about this use, and that these concerns cannot be fully responded to using the traditional methods of philosophy. We need an understanding of how intuitions are generated and what it is they are based on, and this understanding must be founded on the psychological investigation of the mind. I explore how a psychological understanding of intuitions is likely to impact a range of philosophical projects, from conceptual analysis to the study of (non-conceptual) "things themselves" to experimental philosophy.
\end{abstract}

Keywords: intuitions, psychology, experimental philosophy, conceptual analysis

Philosophers use intuitions when doing philosophy. Not exclusively, not always, and perhaps not all philosophers, but most of us and quite often. Intuitions in many cases play the role that observation does in science-they are the data that must be explained, the confirmers or the falsifiers of theories. However, unlike observation in science, there is widespread controversy about the role intuitions play in philosophy. Robert Cummins (1998), for example, argues that they are "epistemologically useless" in part because of concerns about their accuracy (Cummins 1998, 125), and Hilary Kornblith argues that "philosophy cannot live up to its ambitions" if it continues to emphasize the use of intuitions, since, on his view, they merely tell us about our concepts (Kornblith 2006, 11). More traditionally minded philosophers have defended the use of intuitions against these sorts of criticisms. George Bealer and Lawrence BonJour have argued, for example, that intuitions are essential to the practice of philosophy and attempted to defend their accuracy and usefulness on a priori grounds (Bealer 1998, BonJour 1998). So-called experimental philosophers have come down on both sides of this debate. Famously, Jonathan Weinberg, Shaun Nichols, and Steven Stich have claimed

Corresponding author's address: Brian Talbot, Philosophy Department, UCB 232, Boulder, CO 80309-0232, USA. Email: Brian.Talbot@colorado.edu. 
on the basis of experimental results that intuitions about knowledge vary from culture to culture, and thus should not be used as the basis for normative conclusions (Weinberg et al. 2001). Others have claimed that careful use of experimental methods can potentially help us respond to some criticisms of intuitions. ${ }^{1}$

In this paper I will advocate a new approach to this debate. Concerns about the use of intuitions are legitimate and justified, and I argue that they cannot be dismissed using only the $a$ priori methods of traditional philosophy. However, abandoning intuitions on the basis of these concerns is too hasty. Instead, we should improve our understanding of what intuitions are and how they are generated in order to assess what role they can and should play in philosophy. I will argue that intuitions are the results of unconscious processes that can only be understood through psychological investigation of the mind. It turns out that these processes are capable of generating useful and accurate evidence about a number of issues in philosophy, although not necessarily all of them. They are able to tell us not only about our concepts but also in some cases about things themselves-extra-conceptual factsbut proper use of intuitions both in conceptual analysis and as evidence about extra-conceptual facts should be guided by an understanding of psychology. Finally, I will look at how this might impact some of the various projects of experimental philosophy.

\section{Worries about Intuitions}

Whatever position one occupies in the debate about intuitions, it is hard to deny that worries about their use in philosophy are legitimate. Intuitions are called upon to do a lot of work for us: we advance philosophical theories on the basis of their agreement with our intuitions, and plausible and useful theories have been discredited because of conflicts with intuitions. At the same time, we generally give no reasons why one should accept the specific intuitions we use as evidence, there are no widely agreed upon views of the sources of intuitions, and despite the fact that intuitions are a mental phenomenon, philosophers generally have little understanding of the mental processes that affect them. What is more, we know for a fact that intuitions are not a wholly reliable source of evidence; not only can different people have different intuitions about the same case, the intuitions of a single individual can sometimes conflict. There seem to be few clear marks that differentiate trustworthy types of intuitions from untrustworthy, nor do we have any good data on the frequency with which our intuitions are wrong. When so much weight is placed upon a source of alleged evidence that we

\footnotetext{
${ }^{1}$ (Weinberg et al. 2006).
} 
do not understand, and the reliability of which can easily be questioned but not easily checked, it makes sense to be concerned.

One might, however, accept that reasons for concern exist without accepting that these concerns must be responded to. Ernest Sosa (1998) has argued along these lines. He claims that worries about intuitions are similar to those we have about perception; since our use of perceptual evidence is justified despite these worries, our use of intuitions is as well. We certainly know that sometimes our senses mislead us (when observing small objects, or those far away, for example), and we know that our senses might entirely mislead us (if there were an evil demon). In addition, people did not understand how sense perception worked for most of human history. Even so, our use of our perceptual faculties was and remains justified. Why, then, demand that we understand how our intuitions work or be able to assuage worries about their reliability in order to use them as evidence?

Worries about intuitions are more pressing than those about perception. Let us bracket evil demon style skepticism; few of us take the possibility of evil demons as reason to stop using our senses, and worries about intuitions are not of this sort. These worries to the side, we have a good understanding of when sense perception actually goes astray. That is, we can give a systematic account of the conditions in which we are likely to get bad data from our senses (e.g. when we are asleep, or very tired, or have ingested certain chemicals). This prevents facts about failures of our senses from giving rise to general doubts about the use of perception, since our evidence about perceptual error is that (as far as we know) it is limited to an identifiable set of circumstances. We are not in an analogous position with regard to intuitions in philosophy. There are some identifiable classes of intuitions that we know are especially error prone-intuitions about infinite sets are a good example. But we can point to examples of erroneous intuitions throughout philosophy, and these errors do not seem to be limited to specific philosophical domains or topics, nor (as far as we currently know) do they only occur in identifiable circumstances. I know of no attempt to systematize all or the majority of errors in philosophical intuitions, and the lack of a systematic account of intuitive errors puts us in a different, and worse, epistemic position with respect to intuitions than we are in with respect to perception. The proper response to concerns about intuitions is not to try to argue for the status quo - the continued use of intuitions with no understanding of their sources or reliability—but instead should involve determining whether and to what extent intuitions can accurately tell us facts of philosophical interest.

The best way of doing this is to develop a general and systematic understanding of how intuitions work: where they come from, how they are generated, what they are and are not based on, what factors affect them. Such 
an understanding is worth pursuing for a number of reasons. It, combined with an understanding of what kind of evidence we need for our various philosophical projects, could alleviate uncertainty about the usefulness of intuitions, allow us to refine our methods of gathering them, and help us to only use them when they are reliably accurate. Such an understanding may also be helpful in resolving conflicts between intuitions, since some of the conflicting intuitions may turn out to be of an unreliable sort. There are, of course, other ways of learning about the reliability of intuitions, among them checking intuitions against known facts, but there are limits on their applicability that would not affect a general understanding of the sources of intuitions. For example, checking intuitions against known facts to test their reliability will only work to the extent to which we know the answers our intuitions should give, and would not be very useful in philosophical domains about which relatively little is currently known for certain. A general understanding of how intuitions work could be useful in checking the reliability of our intuitions in such domains, however. Even with only a little knowledge about some philosophical topic, we might be able to set standards (at least minimal ones) that something would have to meet to qualify as a source of data about it. Given a general understanding of how intuitions work, we could then determine if they at least meet those standards.

George Bealer and Lawrence BonJour, among others, have accounts of intuitions that aim at giving us a general understanding of how they work. Their accounts are attempts to build theories of intuitions a priori. I argue that a correct understanding of how intuitions work can only be gained empirically and only by doing psychology, not philosophy. As we will see in the next section, this follows in part from the nature of intuitions.

\section{Intuitions and Psychology}

In order to see that psychology is necessary to understand how intuitions work, we first need to know what intuitions are. In colloquial use, 'intuition' refers to a faculty and also to the deliverances of that faculty: we can say 'My intuition tells me P', and also 'I have the intuition that P'. I will use the term only in the second way, in part because that is how the term is used in contemporary philosophy, and also so as not to assume that there is a single faculty of intuition. Intuitions in this sense are had by people; let us call a person who has a given intuition an intuitor. When an intuitor has an intuition, that intuition has some propositional content, and because of this we can say that the intuition is about something (the things that the content represents). So, if Fred has the intuition that murder is wrong, Fred is the intuitor, the content of the intuition is that murder is wrong, and the intuition is about murder and wrongness. 
But what is the intuition? ${ }^{2}$ An intuition is not its content, just as beliefs and desires are not identical to their contents. An intuition is a kind of experience. ${ }^{3}$ George Bealer's term for it, which I think apt, is 'seeming' - an intuition is some content seeming to be true (Bealer 1998). However, not every seeming is an intuition. Intuitions are typically distinguished from what are sometimes called "perceptual seemings", such as the seeming that there is a computer in front of me that is due to my seeing a computer in front of me; from seemings due to recollection, such as the seeming that I have been to Disneyland that is due to my recalling that I have been to Disneyland; and from seemings that are due to beliefs becoming occurrent, such as the seeming that intuitions are seemings that is due to my becoming once again conscious of my belief that this is true.

I want to distinguish intuitions from one other type of seeming, as this distinction is essential to understanding what intuitions are. Sometimes something seems true to one just because one has consciously employed some sort of reasoning and concluded that it is true. For example, imagine I hear an argument, consider each of its premises and come to understand that they are true, and employ truth tables and come to see that the argument is valid. Based only on this, the conclusion of the argument seems true to me. This seeming is not an intuition. This is true in part because this just is not how we use the term 'intuition'. What we call intuitions are things that just strike us as true without us knowing entirely why they do. Even more compellingly, if intuitions were seemings due entirely to conscious reasoning, they would not play the role in philosophy that they do. Intuitions are often used as if they were evidence, so the principle of charity tells us that we should take them to be the sort of thing that could possibly be evidence. If a proposition seems true because we have reasoned about it (and only because of this), the fact that it seems true does not give us any evidence that it is true beyond the evidence upon which we based our reasoning. If we counted the feeling as evidence in addition to the evidence we reasoned from, we would be double counting our evidence (since the feeling comes solely from the evidence used in the reasoning). To make the same point another way, for any proposition that seems true solely on the basis of conscious reasoning, we would have just as much evidence for its truth even if we had reasoned in exactly the same way to the conclusion and it did not feel true. Thus, if some

${ }^{2}$ Here I am trying to draw upon the consensus about intuitions in philosophy; although I disagree with many philosopher's views of how intuitions come about and their exact evidentiary status, I do want to be talking about the same thing as they are. See (Sosa 1998), (Bealer 1998), (Pust 200o), (BonJour 1998), (Cohen 1986), for more detail on the claims in this paragraph.

3 See (Pust 2000) for a discussion of why accounts that allow intuitions to be dispositions, or non-occurrent in some way (thus not necessarily experiences) fail. 
proposition seems to be true and that seeming arises solely from conscious reasoning, the seeming is not evidence for its truth. Since intuitions are supposed to be evidence (at least some of the time), they cannot be based entirely on conscious reasoning (although they are often based partly on it). ${ }^{4}$ That intuitions cannot be based solely on conscious reasoning should not be surprising. Philosophers ought to be interested in a source of evidence that is not based on conscious reasoning, since conscious reasoning often (maybe even always) involves application of theory and we use intuitions to criticize or support theories. The fact that they are not based solely on conscious reasoning makes intuitions seem like a non-question begging source of evidence for and against theories.

This distinction is crucial to the investigation of intuitions. We should be pursuing an understanding of how intuitions come about, what factors affect them, and so forth. Intuitions do not come solely from conscious mental processes, and conscious mental processes are the only ones we have introspective access to; thus, we cannot gain this understanding wholly through introspection. We also cannot figure out how intuitions come about through a priori reasoning alone, since there are a great number of possible unconscious mental processes that could generate seemings of the sort we are discussing. This is not to say that introspection and a priori reasoning are wholly irrelevant to the study of intuitions, or that they cannot tell us anything about them. We can rule out some theories of intuitions a priori (for example, logically impossible ones, or ones that would make intuitions infallible) or on the basis of introspection (for example, those that would produce conscious mental states that we do not experience). However, once we have eliminated all the theories of how intuitions work that we can in this way, we are still left with a number of contenders and must look to other methods. Since introspection and a priori reasoning are the traditional tools of philosophy, we must look outside of philosophy for these methods. Given that intuitions are at least partly mental phenomena, we should learn about them via the rigorous, scientific study of the mind; in other words, an understanding of intuitions should come from psychology (or cognitive science, but I will use these terms interchangeably throughout this paper).

\section{Understanding Intuitions without Psychology}

One might try to avoid this conclusion by claiming that the type of intuitions philosophers are interested in are a subset of the seemings I am calling "intuitions", and that we can know how this subset works without consulting

4 The discussion in this paragraph owes a lot to talks I have had with Brian Bowman and Geoff Georgi. It is also similar to an argument made in (Cohen 1986). 
psychology. For example, both George Bealer (1998) and Lawrence BonJour (1998) give accounts of how intuitions are generated (of varying degrees of completeness) that are not based on psychological research. However, any account of the workings of philosophical intuitions that is entirely $a$ priori will run into a significant problem: while we may be able to show a priori that a certain type of mental event must be produced in a certain way, the claim that the seemings philosophers experience and use as evidence are of that type is an empirical claim. ${ }^{5}$

To illustrate, let us consider Bealer's account of intuitions. Bealer's theory of the workings of our intuitive faculties starts from the claim that our intuitions must be a good source of evidence. His argument for this is based on the following premise: worries about the use of intuitions as evidence are themselves based on intuitions. He goes on to argue that if intuitions are unreliable, then these worries are ill-founded; if intuitions are reliable, then "we have a wealth of concrete-case intuitions to the effect that intuitions are prima facie evidence [...] Because these intuitions about the evidential status of intuitions would be reliable, it would follow that intuitions are in fact prima facie evidence [...]" (Bealer 1993, 107). In either case, the argument goes, we need not be concerned about the evidential status of intuitions.

There are a number of problems with this argument, but I will focus on one only. ${ }^{6}$ Even if intuitions are generally reliable, this does not mean that they are reliable in every circumstance or about every subject-for example, intuitions about intuitions might be unreliable. Further, it does not mean that intuitions about the evidential status of intuitions are correct. So Bealer has not shown that intuitions are prima facie evidence. Nor has he shown that, if intuitions are reliable, we even have evidence that intuitions are evidence; this requires either that ' $\mathrm{X}$ is reliably accurate' entails ' $\mathrm{X}$ is evidence', or that we have evidence that intuitions are reliable. Bealer's argument about the evidential status of intuitions requires that we know something about how intuitions are produced and when they do and do not go wrong. It cannot, then, be used as the basis for an account of how intuitions work.

BonJour's account of intuitions runs into a similar problem. Like Bealer, BonJour bases his account of how intuitions work on claims about epistemology. Specifically, he argues that to avoid skepticism we must have a source of a priori justification, and that the only one that will do is intuition;

5 This is not true across the board; if one consequence of a theory of how intuitions work is that they are always accurate, for example, we can show that our intuitions are not produced in that way without consulting psychology. But for any viable theory of intuitions, showing that it applies to our intuitions is still a largely empirical project.

${ }^{6}$ See (Cummins 1998) especially footnote 8 , for a response to the claim that worries about intuitions are based on intuitions and self defeating. 
he then goes on to explain how intuitions could work in order to avoid skepticism. Even granting BonJour's claims about skepticism and $a$ priori justification, he has not given a strong foundation for a general theory of the sources of intuitions. The connection BonJour brings up between intuitions and skepticism is that avoiding skepticism requires a priori justification in employing certain reasoning methods. All that needs to be true about our intuitions to avoid the skeptical problems BonJour raises is that some intuitions about certain reasoning methods give us justification for using these methods. ${ }^{7}$ There are a number of ways that intuitions could be generated so that they would justify use of these reasoning methods, but unfortunately many of these differ in how much justification we would get from intuitions they generate on other topics. One can fairly easily conceive of ways of generating intuitions that give us justified beliefs about reasoning methods but vary widely with regards to, for example, the justificatory status of the moral or metaphysical intuitions they produce. ${ }^{8}$ So even if BonJour is right about the connection between intuitions and skepticism, the question of how we generate our intuitions remains open and interesting, and the answer will be contingent and a posteriori.

What lesson does this illustrate? Developing a priori a theory of the sources of the intuitions that philosophers experience and use as evidence is not going to produce a very useful theory. Too much about these seemings is, due to their nature, hidden and not amenable to philosophical scrutiny. Because of this, there are a great number of importantly different theories that fit what we know a priori (or due to introspection) about philosophical intuitions. In order to determine how intuitions work, we need more facts, facts that will come from psychology.

One final point: philosophers should not be only interested in psychological studies of the intuitions we use in philosophy (intuitions about philosophical topics like good and bad, knowledge and justification, substance and mind, and so forth). We should be very interested in studies of ordinary, every-day intuitions-intuitions about the categorization of animals and household objects, or the possibility of ordinary events occurring, or intuitions about the ordinary behavior of other human beings. To see why this is, consider one source of serious worry about intuitions in philosophy: that we do not know the extent to which they are reliable. Robert Cummins argues that if we could address concerns about the reliability of intuitions in

7 See (BonJour 1998, chapter 1).

${ }^{8}$ For a very crude example, compare the following two sources of intuitions: the first is whatever source you like that gives one justified beliefs about any philosophical issue. The second is that same source except with a "mental block" when (and only when) it comes to producing intuitions about ethical questions. 
philosophy, then we would not actually need to use intuitions as evidence (Cummins 1998). His argument is that in order to know whether or not intuitions are reliable sources of data we need to determine the extent to which (and the conditions in which) they get us the correct answers to questions. However, if we can do this, then we must have a source of correct answers to philosophical questions that is not based on intuitions, and thus we do not need intuitions. So, he argues, either we cannot know intuitions are reliable (and thus should not use them) or we do not need to use intuitions. Cummins has, however, overlooked one way of checking the reliability of our intuitions. We can determine how intuitions work-the data they are likely to be sensitive to and the data they are likely to ignore, and what factors make them more or less accurate-by studying intuitions about non-philosophical questions we know the correct answers to. These are questions about ordinary objects, behavior, possibilities, and so forth. We can compare what we learn about how intuitions do and do not work for ordinary questions with our demands on a source of evidence for philosophical questions, and calibrate our intuitions in this way. But this means that we need to inform ourselves about the workings of intuitions about somewhat prosaic topics. ${ }^{9}$

One might wonder about how an understanding of intuitions based in psychology would intersect with different types of philosophical projects. Is it really of relevance to every type of philosophical inquiry? Are there domains about which we can determine, without psychology, that intuitions just cannot tell us anything interesting, or those about which we can put concerns to the side without looking to psychology? In the next three sections, I will consider these questions by discussing conceptual analysis, the study of extra-conceptual facts (facts about things other than concepts), and experimental philosophy.

9 One worry about this claim comes from the theory that the mind is modular-that judgments on specific topics are generated by parts of the mind devoted to that topic alongand that learning about how intuitions about a given topic are generated would give us limited or no insight into how intuitions about different topics come about. This is only a worry if differences in how judgments on different topics are generated are largely innateif the processes used by mental modules to generate judgments are almost entirely the product of genetics. It could be, though, that mental modules are the result of one, or a few, general learning processes that build them over our lifetimes. If so, we could learn how various modules work by learning how the general module-building process works. A large number of theorists who take the mind as modular do not accept that these modules are innate, and I think the evidence for innateness is quite weak, but this debate is outside the scope of this paper (for more, see Carruthers 2006). 


\section{Do We Need Psychology to Do Conceptual Analysis?}

One view of the role of intuitions in philosophy is that they help us to do conceptual analysis. By examining one's intuitions about $\mathrm{X}$ one gains a better understanding of one's concept of X. Conceptual analysis is an important step on the road to learning about $\mathrm{X}$, since it is difficult to answer questions about something when one does not know what it is the questions are about. For example, if I want to answer the question 'Do I have free will?' it is very important for me to know what my concept of free will involves, which requires me to do conceptual analysis, which requires that I use my intuitions. This is a relatively modest view of the role of intuitions (to borrow a phrase from Jackson 1998); according to it, intuitions need not reflect anything about the world outside of our heads. Conceptual analysis is generally seen as a prototypical armchair project, one for which the traditional tools of philosophy are sufficient (see, e.g., Fumerton 1999). Given this, we would expect many conceptual analysts to take psychological findings on intuitions to be irrelevant to their project. They should not, however, because intuitions about a subject matter do not always reflect one's concept of that subject matter, and when they do reflect one's concepts, they often do so in a less than straightforward way. Determining the relationship between our intuitions and our concepts requires that we understand how intuitions are produced. ${ }^{10}$

Let us look at some examples. In the right conditions, an intuition with some content can be caused simply by recently hearing or seeing a sentence with the same or related propositional content, even if the intuitor was given no evidence that that sentence is true, and sometimes even if they were told that the sentence is false (Gilbert 1991, Gilbert et al. 1990, 1993, Begg et al. 1992). An intuitor thus might have the intuition that "one cannot try to do something without intending to do it", due not to that intuitor's concepts of trying or intending, but rather due to having heard that statement recently made. ${ }^{11}$ Intuitors can also interpret thought experiments in surprising ways without knowing that they are doing so. This can cause a philosopher to form an erroneous view of the concept the thought experiment is supposed to give information about. For example, people have what is called a hindsight bias. This bias causes them, once they know the outcome of an action or event, to believe that the outcome was more or less inevitable, and that prior to the outcome people could have known or did know that the outcome was inevitable (Schwartz and Vaughn 2002). This is likely to affect intuitions about

${ }^{10}$ This argument is developed more in my paper "The End of the Armchair for Conceptual Analysis?" (manuscript).

${ }^{11}$ This example was picked more or less at random, and I mean to cast no aspersions on any who actually have that intuition. 
moral luck. In many thought experiments about moral luck, two agents perform the same action, but the results of their actions are different. Intuitively, they are differently praise- or blameworthy. This is supposed to show us that our concepts of praiseworthiness and blameworthiness are such that a person can be properly praised or blamed for things outside their control. However, hindsight bias may cause intuitors to unconsciously "believe" that the agents in the thought experiment knew (or should have known) how their acts would turn out. Thus, the differential praise or blame may be due to seeing the agents as acting differently, one with the foreknowledge that things will turn out well, the other with the foreknowledge that things will turn out ill. This does not generate a moral luck problem. We can discover whether or not this bias affects our intuitions, and potentially control for it when it does, through rigorous study; however, this possibility is not one that we would be aware of without an understanding of psychology. This shows that conceptual analysis via intuitions is not always straightforward and it may be quite easy to go wrong without an understanding of our psychology.

Psychology can not only affect the way we interpret our intuitions, but also the shape of analyses of concepts based on intuitions. ${ }^{12}$ Since at least Wittgenstein, philosophers have been aware of how difficult it is to analyze concepts into sets of a certain kind of necessary and sufficient conditions. Further, psychologists have produced evidence that, if our intuitions are an important guide to the shapes of our concepts, then for many concepts no list of necessary and sufficient conditions of the kind philosophers are interested in can be given. ${ }^{13}$ However, there is also psychological research that suggests that some types of concepts are more likely than others to be amenable to analysis into philosophically interesting necessary and sufficient conditions; these are what are called "basic level" concepts, which are less likely to be Wittgensteinian family resemblance categories than so-called "superordinate" concepts (for more on basic level categories, see Mervis and Rosch 1981, Rosch and Mervis 1975, Murphy 2002). If it turns out that basic level concepts are more often analyzable into necessary and sufficient conditions using intuitions than non-basic level concepts, then the methods used to analyze a given concept, and the sorts of results we expect to produce, should vary based on whether or not the concept in question is basic level; identi-

${ }^{12}$ I am not by any means the first to notice this. See, for example, (Ramsey 1998).

${ }^{13}$ The most famous discussion of this topic is from (Rosch and Mervis 1975), but controversy about their results and what they mean is ongoing. It may be, for example, that concepts are better captured by looking at a combination of intuitive and reflective use of words, so that we can give necessary and sufficient conditions for application of a concept as long as we look to data from non-intuitive use of the concept. Further, this is also not to say that no list of necessary and sufficient conditions can capture these concepts, but rather that these lists will look quite different than those philosophers are typically interested in. 
fying concepts as basic level requires an understanding of psychology.

My goal here is not to list every way in which psychological research bears on the use of intuitions for conceptual analysis, but rather to show that it can and does in some ways, in order to show that it is important for us to start figuring out what those ways are. We can see, then, that even if one's interest in intuitions is due only to an interest in conceptual analysis, one ought to pay attention to psychological research.

\section{Intuition and "Things Themselves"}

We have just seen some ways that an understanding of psychology is important to the use of intuitions for conceptual analysis. Many of us, however, are interested in more than simply what our concepts of things like responsibility or intention or causation are-we want to know what responsibility, intention, or causation themselves actually are. ${ }^{14}$ Some philosophers, such as Hilary Kornblith or Frank Jackson, interested in "things themselves" rather than our concepts of them, have argued that intuitions should play at most a very limited role in philosophy (e.g. Kornblith 2006 or Jackson 1998). They argue that we might look to intuitions to understand very generally what the subject of some domain of philosophy (such as epistemology or metaphysics) is, or how we ought to talk about it, but once we have done that intuitions are no longer useful because they do not tell us facts about things themselves.

This conclusion is not always warranted. Psychology shows us that intuitions can tell us about more than just the content of our concepts, and that they can tell us things we would be hard pressed to discover without them. However, intuitions will not necessarily be useful evidence about every question in philosophy. Philosophers interested in facts about "things themselves" should thus be interested in a psychologically-based understanding of how our intuitions are generated and what information they are based on. Intuitions can potentially be an extremely helpful tool, and to understand when and how to use this tool we need to understand their source.

To see this, let us consider some facts about the capabilities of our unconscious minds, since intuitions are generated in part by unconscious mental processes. The unconscious is superior to the conscious mind at process-

${ }^{14}$ Hilary Kornblith, for example, says

My own view is that our concepts of knowledge and justification are of no epistemological interest. The proper objects of epistemological theorizing are knowledge and justification themselves, rather than our concepts of them. (Kornblith 2006, 11-12). 
ing information in some ways. In fact, when we use conscious faculties to make judgments that are usually made unconsciously, the results are often inferior to what the unconscious mind would have produced (e.g., Wilson and Schooler 1991). Our unconscious minds can track relationships that occur over longer periods of time, or are more complex, than our conscious minds can track without mechanical assistance (Lewicki et al. 1992). Our unconscious is also sensitive to information that our conscious minds will not normally notice. Our unconscious detects, processes, and makes judgments based on information that we consciously consider irrelevant (e.g., Lewicki et al. 1989, Betsch et al. 2001). This is important because this information can actually be relevant to judgments without our knowing it. Our unconscious can use information for which we have no words, whereas this can be quite difficult for our conscious minds. ${ }^{15}$ The unconscious is less sensitive to distraction and to other mental demands than is the conscious mind-it keeps noticing and processing information even when the conscious mind is overwhelmed (e.g. Betsch et al. 2001, Dijksterhuis 2004, Gilbert and Krull 1988). Some specific examples of judgments that the unconscious excels at making are social judgments and judgments about our own mental states: we are able to ascertain the feelings of others, predict their behavior, and judge when they are honest without knowing how we do so (Ambady and Rosenthal 1992), and our unconscious has access to information about certain of our own beliefs, desires, motivations, and opinions that our conscious mind has no direct access to (Wilson 2002).

What does all this tell us? It tells us that many of our intuitions will be based on information we would not consider using, or be able to use, consciously. This information can be the sort of information we ought to be paying attention to if we wish to make accurate judgments about philosophical topics. To see this, consider three related philosophical subjects: responsibility, intention, and causation. Attributions of responsibility, or intentionality, or causality, should be sensitive to subtle distinctions between people, or mental states, or physical relationships. Both causation and responsibility come in degrees, and the amount of each which should be attributed most likely depends on a multitude of small and easily overlooked factors present in different situations. The ability to make accurate judgments about these three should require the ability to put together vast amounts of minute details and notice patterns that occur over long periods of time. These are exactly the sorts of things our unconscious mind is capable of doing better than our conscious mind. Thus, we have reason to think that in some cases intuitions about responsibility, intention, or causation will be based on real and important distinctions that we would be likely to overlook using only our

\footnotetext{
${ }^{15}$ See for example (Murphy 2002), or research on infants such as (Campos et al. 1978).
} 
conscious faculties. Parallel arguments can plausibly be made for numerous topics in metaphysics, ethics, epistemology, the philosophy of action, and the philosophy of mind.

This argument is of necessity something of a promissory note. My point is not that our intuitions will always give us useful information about things themselves, a claim that would be hard to assert given the obvious fact that intuitions are not always accurate. My point is that we have reason to expect that they can in some cases give us such information, and that this information may be difficult to obtain otherwise. A developed, empiricallyinformed understanding of our intuitive faculties of the sort I have been arguing for in this paper is needed to determine which cases these are, and to allow us to best use intuitions when they can be helpful.

\section{Experimental Philosophy}

Experimental philosophy is the recent movement to incorporate experimental rigor into the gathering of intuitions. Some experimental philosophers believe that intuitions are a philosophically important source of evidence, while others have employed experimental results to cast doubt on their use. We should be hesitant to accept the results of philosophical experiments if they are not supplemented by an understanding of the psychology behind our intuitions.

To make this point more clearly, let us consider an example, one of the most famous pieces of experimental philosophy: the work of Jonathan Weinberg, Shaun Nichols, and Stephen Stich on epistemic intuitions (2001). They advance the following claims: intuitions about knowledge and justification tend to vary from culture to culture, and this undermines our reasons for using intuitions about knowledge and justification as evidence for epistemic theories. They argue that when intuitions about epistemology differ between groups, we have no good reason to choose one set of intuitions as evidence over the other. If we have no principled way to choose between the intuitions, and intuitions are supposed to be the basis for our theory, we have no way to choose between a theory based on one set of intuitions or the other. It is inappropriate to choose a philosophical theory arbitrarily, or based on provincial considerations (e.g., we are more accustomed to one theory), especially when that theory is normative, as are theories of justification or knowledge. Thus, differences in intuitions about epistemology between groups are problematic for those who wish to found their theories on intuitions.

The evidence Weinberg, Nichols, and Stich give that there are cultural variations in intuitions comes from experiments in which they presented versions of various classical epistemological thought experiments (such as 
Gettier cases) to subjects from different cultural backgrounds, specifically East Asians and Westerners, and to subjects of different socio-economic status. They found statistically significant variations in reactions to some of these thought experiments. From this, they concluded that there are differences in intuitions between the groups, and thus that basing theories of knowledge or justification on intuitions is problematic.

One plausible objection to this conclusion comes from Ernest Sosa (2005). Sosa makes the point that, "[g]iven that these subjects are sufficiently different culturally and socio-economically, they may because of this import different assumptions [...]" as they consider the situations given in these thought experiments, with the result that they are not in actuality disagreeing (Sosa 2005, 107). This point, however, is speculative-these subjects may be interpreting the thought experiments differently, but we have no particularly strong evidence that they are. This is where a psychologically informed understanding of intuitions plays a vital role; the best way to assess Sosa's objection would be to look for empirical evidence that there are or are not cross cultural differences in interpretations of these thought experiments. Consideration of psychological findings on cultural cognitive differences gives us just such evidence.

According to Weinberg, Nichols, and Stich, "Richard Nisbett and his collaborators have shown that there are large and systematic differences between East Asians and Westerners on a long list of basic cognitive processes including perception, attention and memory" (Weinberg et al. 2001). The existence of differences in cognitive processes between the groups Weinberg, Nichols, and Stich tested makes less speculative the claim that such differences may have caused differences in interpretation of the thought experiments used. It also opens up another possible objection to their argument: the processes that generate one culture's intuitions may be systematically better at generating the intuitions in question, or one group may be prone to a bias that makes their intuitions less relevant to epistemology, and this would give us a means of resolving the conflict. Although I do not have the space to do an exhaustive review of Weinberg, Nichols, and Stich's results given findings on cultural cognitive differences, by considering some examples I can show that it plausible that the discovered intuitive differences are due to cultural cognitive differences, and thus show that their research (and experimental philosophy more generally) would benefit from further examination of the psychology behind our intuitions.

According to the researchers cited by Weinberg, Nichols, and Stich, East Asians are supposed to be more subject to hindsight bias than Americans, whereas American subjects are more likely than East Asians to make what 
is called the "fundamental attribution error" (Norenzayan et al. 2002). ${ }^{16}$ As discussed above, hindsight bias is the tendency, once one knows how something turns out, to think that that outcome was more or less inevitable and predictable from the outset. The fundamental attribution error is the tendency to "make inferences about the dispositions of others even when situational forces explain the behavior quite nicely" (Gilbert 2002, 169). For example, in one study, "students who are randomly assigned to receive bad news may, on average, be judged as more chronically depressed than students who are randomly assigned to receive good news" (Gilbert 2002, 169). These differences could explain differential reactions to thought experiments such as Gettier cases.

What is typical of a Gettier case is that a person is described who uses a belief forming method (such as deductive reasoning) which normally is a good one to use; they end up forming a true belief, but because of details about their situation, this is due largely to luck. Westerners were more likely than Asians to say that person described in Gettier cases only believed something, rather than knew it. Note that in Gettier cases there is a factbelieving something true due to luck-which is perfectly well explained by a quirk of the situation the believer finds themselves in. A person prone to the fundamental attribution error, and thus likely to make character judgments based on single events, might explain this fact however as due to the character of the believer and think that the person described normally relies on luck in their reasoning. How might this affect their intuitions? It is plausible that the ways in which one typically reasons and forms beliefs makes a difference as to whether or not one knows any given proposition they believe; this is the core of virtue epistemology. If we are tacit virtue epistemologists, then seeing someone as a person who normally relies on luck to form their beliefs would give us reason to think that a specific belief they formed in this way would not count as something they know. However, if we saw that person as someone who did not normally rely on luck to form true beliefs, then the fact that luck played a role in this case might not entail a lack of knowledge (in this case). Thus, Westerners' greater tendency to say that people described in Gettier cases do not know what they believe makes more sense given their greater tendency to commit the fundamental attribution error.

Likewise, cultural differences in intuitions about Gettier cases might also be due in part to hindsight bias. This bias makes people prone to think that the way things actually turned out was inevitable and predictable from the onset. In Gettier cases, one comes to form a true belief through luck; how-

\footnotetext{
${ }^{16}$ Weinberg, Nichols, and Stich cite (Nisbett et al. 2001), whereas I am citing (Norenzayan et al. 2002). However, the two papers share two co-authors, and both refer to similar bodies of research.
} 
ever, if coming to this true belief was inevitable, or predictable, then it looks less like the product of luck. If the role of luck is part of why the believer in Gettier cases fails to know what they believe, as many philosophers claim, then it makes sense that people who see luck as less of a factor in the situation described also tend to think that the believer in question really knows what they believe. Asians' greater tendency to experience hindsight bias might partly explain why they have a greater tendency to intuit that people in Gettier cases really do know what they believe.

This is only the sketch of an argument, and more research-both philosophical and empirical-is needed. It is unlikely that either factor by itself explains all of the differences in responses found by Weinberg, Nichols, and Stich; cross-cultural differences in the tendency to commit the fundamental attribution error, for example, are not as large as the differences in responses detected. ${ }^{17}$ My goal, though, is not to refute Weinberg, Nichols, and Stich's arguments, but to illustrate the following point: the success of their argument turns on psychological facts, facts about how thought experiments are interpreted, and about the presence or absence of mental biases that could discount the normative intuitions of some groups. Thus, a full evaluation of their arguments calls for an understanding of the psychology of intuitions. We can extrapolate from this example to experimental philosophy in general, since even when experimental philosophers do not study normative phenomena, their arguments still require an understanding of how intuitors generate their intuitions. Changes in the way we gather intuitions of the sort advocated by experimental philosophers should go hand in hand with the application of the sort of understanding of intuitions that I am advocating.

\section{Conclusion}

Philosophers have good reason to be concerned about the use of intuitions in philosophy. To address these concerns, we should have an understanding how our intuitive faculties work, what intuitions are based on, and what might make them go awry. Because intuitions cannot be generated by conscious mental processes, but can be generated by any number of unconscious ones, we cannot gain such an understanding just by doing philosophy (that is, purely a priori or through introspection). We need to look at what psychologists have learned about intuitions. A psychologically informed theory of intuitions is of relevance to most philosophers, whether they are interested in conceptual analysis or whether they are interested in "things themselves".

${ }^{17}$ A defense of Weinberg, Nichols, and Stich's results along these lines actually bolsters my claim about the importance to experimental philosophy of an understanding of how our intuitions work, since such defenses are based themselves in an understanding of psychology. 
This does not mean that psychology can replace philosophy, or that it will answer philosophical questions by itself. Theory building in the light of intuitive evidence (or the lack thereof), and thinking about what questions to ask and how to ask them, are philosophical, not psychological, tasks.

\section{Acknowledgements}

I would like to thank Steve Finlay, Janet Levin, Kadri Vihvelin, Julia Staffel, Brian Bowman, and Geoff Georgi for their help with this paper.

\section{Bibliography}

Ambady, N. and Rosenthal, R. (1992). Thin slices of expressive behavior as predictors of interpersonal consequences: A meta-analysis, Psychological Bulletin 111: 256-274.

Bealer, G. (1993). The incoherence of empiricism, in S. J. Wagner and R. Warner (eds), Naturalism: A critical appraisal, University of Indiana Press, Notre Dame, pp. 163-196.

Bealer, G. (1998). Intuition and the autonomy of philosophy, in M. DePaul and W. Ramsey (eds), Rethinking intuition, Rowman \& Littlefield, Lanham, pp. 201-240.

Begg, I., Anas, A. and Farinacci, S. (1992). Dissociation of processes in belief: Source recollection, statement familiarity, and the illusion of truth, Journal of Experimental Psychology: General 121: 446-458.

Betsch, T., Plessner, H., Schwieren, C. and Gutig, R. (2001). I like it but i don't know why: A value-account approach to implicit attitude formation, Personality and Social Psychology Bulletin 27: 242-253.

BonJour, L. (1998). In defense of pure reason, Cambridge University Press, Cambridge.

Campos, J. J., Hiatt, S., Ramsay, D., Henderson, C. and Svejda, M. (1978). The emergence of fear on the visual cliff, in M. Kewis and L. Rosenblum (eds), The development of affect, Plenum, New York, pp. 149-182.

Carruthers, P. (2006). The case for massively modular models of mind, in R. Stainton (ed.), Contemporary debates in cognitive science, Blackwell, Malden, pp. 3-21.

Cohen, L. (1986). The dialogue of reason, Clarendon Press, Oxford.

Cummins, R. (1998). Reflections on reflective equilibrium, in M. DePaul and W. Ramsey (eds), Rethinking intuition, Rowman \& Littlefield, Lanham, 
pp. $113-128$.

Dijksterhuis, A. (2004). Think different: The merits of unconscious thought in preference and decision making, Journal of Personality and Social Psychology 87: 586-598.

Fumerton, R. (1999). A priori philosophy after an a posteriori turn, Midwest Studies in Philosophy 23: 21-33.

Gilbert, D. (1991). How mental systems believe, American Psychologist 46: 107-119.

Gilbert, D. (2002). Inferential correction, in T. Gilovich, D. Griffin and D. Kahneman (eds), Heuristics and biases: The psychology of intuitive judgment, Cambridge University Press, Cambridge, pp. 167-184.

Gilbert, D. and Krull, D. (1988). Seeing less and knowing more: The benefits of perceptual ignorance, Journal of Personality and Social Psychology 54: 193-202.

Gilbert, D., Krull, D. and Malone, P. (1990). Unbelieving the unbelievable: Some problems in the rejection of false information, Journal of Personality and Social Psychology 59: 601-613.

Gilbert, D., Tafarodi, R. and Malone, P. (1993). You can't not believe everything you read, Journal of Personality and Social Psychology 65: 221-233.

Jackson, F. (1998). From metaphysics to ethics: A defense of conceptual analysis, Oxford University Press, Oxford.

Kornblith, H. (2006). Appeals to intuition and the ambitions of epistemology, in S. Hetherington (ed.), Epistemology futures, Oxford University Press, Oxford, pp. 10-25.

Lewicki, P., Hill, T. and Czyzewska, M. (1992). Nonconscious acquisition of information, American Psychologist 47: 796-801.

Lewicki, P., Hill, T. and Sasaki, I. (1989). Self-perpetuating development of encoding biases, Journal of Experimental Psychology: General 118: 323-337.

Mervis, C. and Rosch, E. (1981). Categorization of natural objects, Annual Review of Psychology 32: 89-115.

Murphy, G. (2002). The big book of concepts, MIT Press, Cambridge.

Nisbett, R., Peng, K., Choi, I. and Norenzayan, A. (2001). Culture and systems of thought: Holistic versus analytic cognition, Psychological Review 108: 291-310.

Norenzayan, A., Smith, E., Kim, B. and Nisbett, R. (2002). Cultural preferences for formal versus intuitive reasoning, Cognitive Science 26: 653-684.

Pust, J. (2000). Intuitions as evidence, Routledge, New York. 
Ramsey, W. (1998). Prototypes and conceptual analysis, in M. DePaul and W. Ramsey (eds), Rethinking intuition, Rowman \& Littlefield, Lanham, pp. $161-178$.

Rosch, E. and Mervis, C. (1975). Family resemblances: Studies in the internal structure of categories, Cognitive Psychology 7: 573-605.

Schwartz, N. and Vaughn, L. (2002). The availability heuristic revisited: Ease of recall and content of recall as distinct sources of information, in T. Gilovich, D. Griffin and D. Kahneman (eds), Heuristics and biases: The psychology of intuitive judgment, Cambridge University Press, Cambridge, pp. 103-119.

Sosa, E. (1998). Minimal intuition, in M. DePaul and W. Ramsey (eds), Rethinking intuition, Rowman \& Littlefield, Lanham, pp. 257-270.

Sosa, E. (2005). A defense of the use of intuitions in philosophy, in D. Murphy and M. Bishop (eds), Stich and his critics, Blackwell, Oxford, pp. 101112.

Weinberg, J., Crowley, S., Gonnerman, C., Swain, S. and Vandewalker, I. (2006). Intuition and calibration. manuscript.

Weinberg, J., Nichols, S. and Stich, S. (2001). Normativity and epistemic intuitions, Philosophical Topics 29: 429-460.

Wilson, T. (2002). Strangers to ourselves: Discovering the adaptive unconscious, Belknap Press, Cambridge.

Wilson, T. and Schooler, J. (1991). Thinking too much: Introspection can reduce the quality of preferences and decisions, Journal of Personality and Social Psychology 6o: 181-192. 